\title{
Multimodal Imaging and Soft X-ray Nanotomography to Optimize Algal-based Lipid Feedstocks
}

Chuck Smallwood ${ }^{1}$, William Chrisler ${ }^{1}$, Jian-Hua Chen ${ }^{2}$, Emma Patello ${ }^{1}$, Rosanne Boudreau ${ }^{2}$, Mark Le Gros $^{2}$, Gerry McDermott ${ }^{2}, \&$ James E. Evans ${ }^{1}$

${ }^{1 .}$ Environmental Molecular Sciences Laboratory, Pacific Northwest National Laboratory, Richland, WA, USA

2.The Advanced Light Source, Lawrence Berkeley National Laboratory, Berkeley, CA, USA

Oleaginous microorganisms provide opportunities for bioproduction of lipid feedstocks with downstream biofuel applications [1-4]. However, relatively few organisms have been categorized as high-lipid content producers. Therefore, exploration of new metabolic hacking pathways, or discovery of novel oleaginous strains, is of prime interest and may reveal innovative tactics that can be exploited to combat increasing energy demands. Phototrophs are especially attractive bioproduction systems as they utilize carbon dioxide and sunlight to produce energy dense products such as triacylglycerols (TAGs) lipids in a carbon neutral manner. Ostreococcus tauri is an ancient green alga that is the smallest freeliving eukaryote and is found ubiquitously in oceans worldwide [5]. Despite its lineage, abundance and proclivity for thriving in contaminated seawater, the potential relevance of this simple phototroph for bioenergy feedstock applications has remained unexplored.

We utilized an array of multimodal techniques at the single-cell and population levels to characterize nutrient conditions that induce cellular phenotypic variations resulting in the accumulation of triacylglycerol rich lipid bodies in O. tauri. Cryogenic soft x-ray nanotomography allowed us to visualize and compare the intercellular distribution of starch and lipid bodies label-free. Upon observing structural and phenotypic differences of inorganic carbon utilization, intercellular long chain lipids were confirmed and quantified by lipidomics [6]. In this presentation we will describe our coupling of multimodal imaging and systems biology approaches and highlight several nutrient conditions that significantly elevated biofuel relevant lipid feedstock production. We will also discuss current and future work focused on engineering this microorganism to further enhance its productivity and applicability for biofuel applications.

References:

[1] M.H. Liang, J.G. Jiang, Prog Lipid Res 52 (2013), p. 395-408.

[2] I.R. Sitepu, et al, Biotechnol Adv 32 (2014), p. 1336-1360.

[3] S.P. Slocombe et al, Sci Rep 5 (2015), p. 9844.

[4] J. Levering, J. Broddrick, K. Zengler, Curr Opin Biotechnol, 36 (2015), p. 32.

[5] S. Robbens et al, Mol Biol Evol, 24 (2007), p. 956.

[6] A portion of the research was performed using the Environmental Molecular Sciences Laboratory (EMSL), a national scientific user facility sponsored by the Department of Energy's Office of Biological and Environmental Research and located at PNNL. Work was supported by DOE-BER Mesoscale to Molecules Bioimaging Project \#66382. 


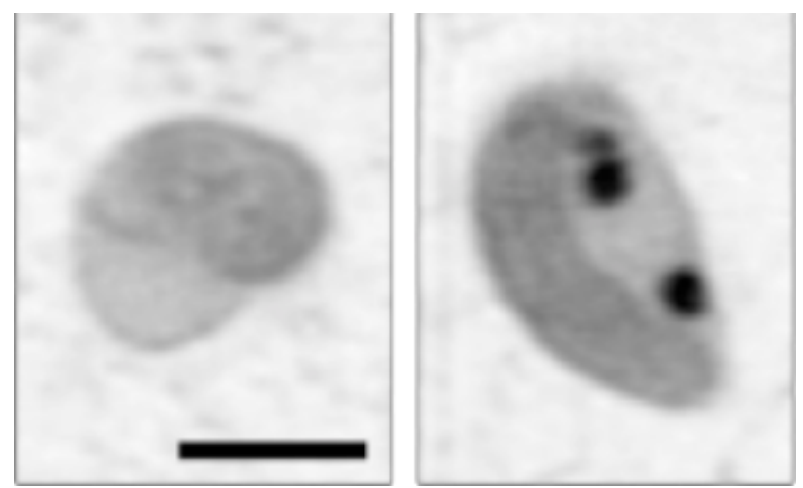

Figure 1. Cryogenic soft X-ray nanotomography of normal O. tauri cell (left) and starved cell (right) showing accumulation of lipid droplets (black circles). Scale bar is equivalent for both panels and represents 1 micron. 